\title{
LDH-intercalated D-gluconate: generation of a new food additive-inorganic nanohybrid compound
}

\begin{abstract}
Intercalation of d-gluconate into the interlamellae of zinc-aluminum-layered double hydroxide for the formation of a food additive-inorganic layered nanohybrid was accomplished by both direct (co-precipitation) and indirect (ion-exchange) methods. Powder X-ray diffraction (PXRD) together with CHNS and Fourier transform infrared (FTIR) analyses showed that the hybridization of d-gluconate with pure phase and good crystallinity was successfully accomplished by a direct method within ranges of $\mathrm{pH} 7.5-10, \mathrm{Zn}$ to $\mathrm{Al}$ initial molar ratio of $2-5$ and DG concentration of $0.05-0.3 \mathrm{M}$. The same nanohybrid compound was also prepared using an indirect ion-exchange method by contacting the preprepared LDH with $0.1 \mathrm{M}$ DG for $80 \mathrm{~min}$. The basal spacing of the nanohybrid synthesized by the direct method ranged between 9 and $12.0 \AA$ while that synthesized by the indirect ionexchange method was $14.0 \AA$. The crystallinity of the latter was higher than the former and it inherited the crystallinity of the precursor. This work shows that a food additive, such as dgluconate, can be hybridized into an inorganic host for the formation of a new nanohybrid compound, which can be used to regulate the release of acidity in the food industry.
\end{abstract}

Keyword: Nanostructure; Inorganic compound; Chemical synthesis; X-ray diffraction 